\title{
Simple Point-Ion Electrostatic Model Explains the Cation Distribution in Spinel Oxides
}

\author{
Vladan Stevanović, Mayeul d'Avezac, and Alex Zunger* \\ National Renewable Energy Laboratory, Golden, Colorado 80401, USA \\ (Received 18 May 2010; published 10 August 2010)
}

\begin{abstract}
The $A_{2} B \mathrm{O}_{4}$ spinel oxides are distinguished by having either a normal $(N)$ or an inverse $(I)$ distribution of the $A, B$ cations on their sublattices. A point-ion electrostatic model parametrized by the oxygen displacement parameter $u$ and by the relative cation valencies $Z_{A}$ vs $Z_{B}$ provides a simple rule for the structural preference for $N$ or $I$ : if $Z_{A}>Z_{B}$ the structure is normal for $u>0.2592$ and inverse for $u<$ 0.2578 , while if $Z_{A}<Z_{B}$ the structure is normal for $u<0.2550$ and inverse for $u>0.2578$. This rule is illustrated for the known spinel oxides, proving to be $\sim 98 \%$ successful.
\end{abstract}

PACS numbers: 61.50.Ah, 61.43.-j, 61.66. $-\mathrm{f}$

The $A_{2} B_{\mathrm{O}_{4}}$ spinel oxides form a family of $\sim 120$ compounds [1] spanning a range of physical properties including ferromagnetism [2], coexistence of transparency, and $p$-type conductivity [3], superconductivity [4], and ferroelectricity [5]. The spinel structure consists of facecentered cubic (fcc) lattice of oxygen anions within which $A$ and $B$ cations occupy octahedral and tetrahedral interstitial sites arranged in one of two possible patterns: nor$m a l(N)$ and inverse $(I)$. In the normal spinel structure ( $F d 3 m$ space group) the tetrahedral sites are occupied exclusively by the $B$ cations while the octahedral sites are occupied exclusively by $A$ cations. The inverse spinel structure represents a class of configurations in which tetrahedral sites are occupied exclusively by $A$ cations but the octahedral sites can be occupied by both $A$ and $B$ cations possibly in a random fashion. The $\sim 120$ known oxide spinel compounds are classified experimentally into Normal or Inverse types $[1,6]$. This includes also dual $(D)$ spinels which are classified according to their degree of inversion $\lambda$ (relative concentration of $\mathrm{A}$ on tetrahedral sites) that can be intermediate between $N(\lambda=0)$ and $I$ $(\lambda=1)$. Despite the importance of $N$ versus $I$ cationic distribution there is still no complete agreement on the nature of the physical and chemical interactions responsible for $N$ or $I$ cationic distributions [1,6-12]. Here we offer a deductive approach based on revisiting the previously discredited $[7,13,14]$ classic point-ion electrostatic (PIE) model for $N$ vs $I$ structural preference [10]. We show that this model predicts $N / I$ assignments from the sole knowledge of the relative formal cation valencies $Z_{A}$ vs $Z_{B}$ and a dimensionless anion displacement parameter $u$ [Fig. 1(a)]. Out of 84 spinel oxides for which the $N / I$ distribution and $u_{\text {exp }}$ are known experimentally, only 11 are in disagreement with the PIE model. However, using density functional theory (DFT) calculations we find that $u_{\mathrm{DFT}}$ is rather different from $u_{\text {exp }}$ in nine of these cases $\left(\mathrm{Cr}_{2} \mathrm{HgO}_{4}, \mathrm{Ti}_{2} \mathrm{MgO}_{4}, \mathrm{Ga}_{2} \mathrm{CuO}_{4}, \mathrm{Ga}_{2} \mathrm{NiO}_{4}, \mathrm{Zn}_{2} \mathrm{PdO}_{4}\right.$, $\mathrm{Mg}_{2} \mathrm{PtO}_{4}, \mathrm{Zn}_{2} \mathrm{VO}_{4}, \mathrm{Fe}_{2} \mathrm{VO}_{4}$, and $\mathrm{Na}_{2} \mathrm{MoO}_{4}$ ) and that using $u_{\mathrm{DFT}}$ rather than $u_{\text {exp }}$ as input to the electrostatic model predicts the experimentally observed $N$ vs $I$. This work suggests that for oxide spinels, point-ion electrostatics is sufficient to determine $N$ vs $I$ preference using a very simple rule: Spinel oxides with $Z_{A}>Z_{B}$ are normal if $u>$ 0.2592 and inverse if $u<0.2578$, whereas those with $Z_{A}<$ $Z_{B}$ are normal if $u<0.2550$ and inverse if $u>0.2578$. This rule offers an effortless method to predict the $N$ vs $I$ preference in spinel oxides which is illustrated both for the experimentally known cases and additional ones created by density functional theory, proving to be $\sim 98 \%$ successful.
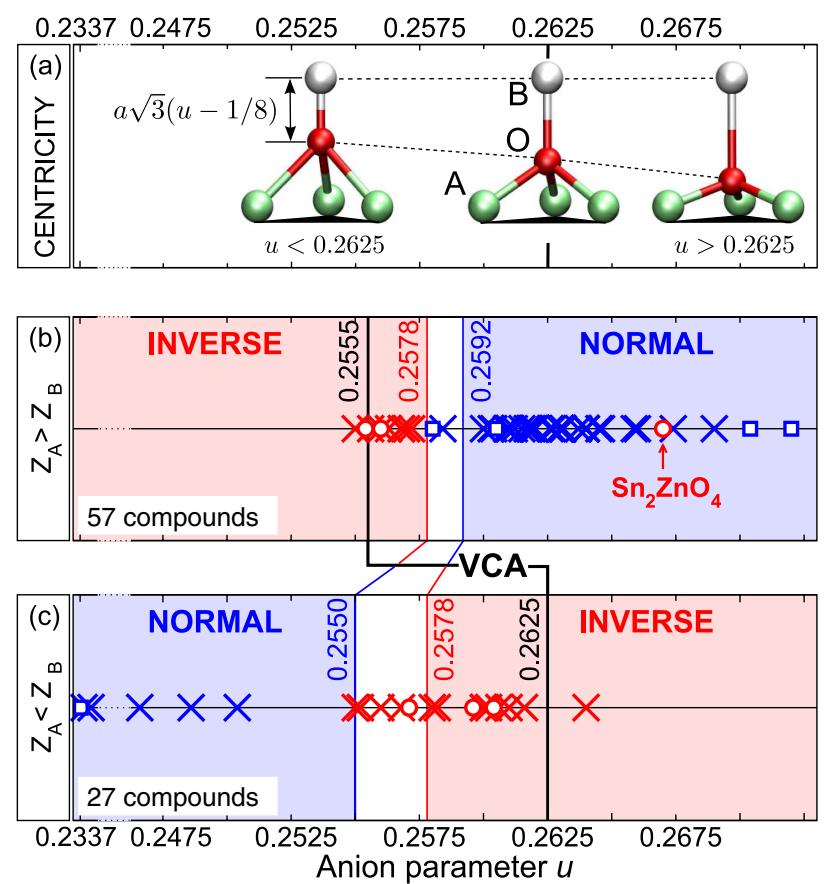

FIG. 1 (color online). (a) The effect of $u$ on the centricity of oxygen anions within their coordination tetrahedra. (b) and (c) The PIE structural maps delineating normal (blue) and inverse (red) structures for: (b) $Z_{A}>Z_{B}$ and (c) $Z_{A}<Z_{B}$ spinels. The critical $u$-regions are shown in white. Crosses represent $u_{\text {exp }}$ for 73 spinel oxides (see supplementary material [16]), colored according to the experimental $N / I$ assignment. For 11 apparent errors of the PIE (Table I) we show only their $u_{\text {DFT }}$, represented as white filled red circles or blue squares depending on the DFT assignment. 
Apparent errors are used, in some cases to detect possible incorrect experimental assignments.

The classic PIE model.-In the point-ion electrostatic model, each $A, B$, and $\mathrm{O}$ atom is given a formal charge $Z_{A}$, $Z_{B}$, and $Z_{\mathrm{O}}$. A similar electrostatic model was used by Bellaiche and Vanderbilt [15] to explain the atomic ordering in complex Perovskite alloys. The atomic positions in the spinel structure are defined by the lattice constant $a$ and a dimensionless anionic displacement parameter $u$. The parameter $u$ describes the centricity of the oxygen position within its coordination tetrahedron as shown in Fig. 1(a): when $u=0.2625$ the oxygen is at the center with equal distances from $A$ and $B$, whereas if $u>0.2625(u<$ $0.2625) \mathrm{O}$ is displaced towards (away from) the $3 \mathrm{~A}$ cations; $u=1 / 4$ places oxygen on the ideal fcc sublattice. The total electrostatic energies of $N$ and $I$ atomic configurations are obtained through the Ewald summation [17]. The inverse spinel consist of a class possible structures. For instance, there are $\left(\begin{array}{c}2 n \\ n\end{array}\right)$ possible inverse spinel configurations in a conventional unit cell (cubic with $n=8$ f.u.). In 1948 Verwey, de Boer, and van Santen [10] represented the whole class of inverse spinels with a single average configuration where octahedral sites are occupied by an average charge $\left(Z_{A}+Z_{B}\right) / 2$, much as within the virtual crystal approach (VCA). They also used the same parameters ( $a$, $u$ ) for both $N$ and $I$. Their VCA-PIE model predicts critical $u$ values delineating $N$ stable from $I$-stable compounds: If $Z_{A}=3$ and $Z_{B}=2$ (called the 3-2 class) the inverse structure had lower Madelung energy for $u<0.2555$, while for $u>0.2555$ the normal structure is more stable. If $Z_{A}=2$ and $Z_{B}=4$ (the 2-4 class) the trend is exactly the opposite with the critical $u_{c}=0.2625$. However, the VCA-PIE model, is not very successful in practice. Indeed, Figs. 1(b) and 1(c) plots $N$ vs $I$ preference for 57 spinel oxides with $Z_{A}>Z_{B}$ (43 belong to 3-2 class) and 27 spinel oxides with $Z_{A}<Z_{B}$ (17 are 2-4) for which $u$ and $N$ vs $I$ preferences were measured (see list in supplementary material [16]). The poor quantitative performance of the classic point-ion electrostatic model was noted, and various corrections, such as Pauli repulsion [13] or crystal field effects $[13,14]$, were offered. However, these models have not lead to more accurate predictions of the cation distribution in spinel oxides [14].

An improved PIE method.-The first obvious improvement is to abandon the VCA and use actual formal charges $Z_{A}$ or $Z_{B}$ on each site, as done in Ref. [7]. Indeed the VCA is a poor approximation also in alloy theory [18] as it ignores fluctuations of the physical quantities. The second improvement is that we distinguish two groups of spinels according to broad inequalities $Z_{A}>Z_{B}$ or $Z_{B}<Z_{A}$. In this way spinel oxides with noninteger formal valencies (e.g., $\mathrm{Mn}_{2} \mathrm{LiO}_{4}$ ) can also be considered and the fact that in reality charges are never exactly formal is also allowed. The third improvement is our finding that for the two broad spinel classes the critical $u_{c}$ delineating $N$ from $I$ is not abrupt, but has a certain width (see the discussion below).
Similar to previous studies $[10,19,20]$ here too we use the same $(a, u)$ for normal and inverse configurations, taking the values from the ground state structure. This is tested here using DFT-calculated volume and position relaxed total energies of different realizations of site occupations of $\mathrm{Al}_{2} \mathrm{MgO}_{4}$ on a 56 atom ( $n=8$ f.u.) unit cell (solid black dots in Fig. 2), compared with point-ion electrostatic energies (red spheres in Fig. 2) calculated with formal ionic charges and $(a, u)$ taken from the normal (ground state) DFT structure. The dielectric constant $\varepsilon$ is used as a scale factor. We see from Fig. 2 that the relative PIE energies of various configurations track well the relative DFT total energies.

Constructing the generalized PIE model and critical $u$ values-We solve for the electrostatic energy of the normal and each of the 2987 inequivalent inverse configurations (constructed with $n \leq 8$ f.u.) using the Ewald summation formula [17] for different values of $Z_{A}, Z_{B}, a$, and $u$. For each choice of $\left(Z_{A}, Z_{B}\right)$ we find $u=u_{c}$ that delineates $N$ from $I$ ( $u_{c}$ does not depend on $\left.a\right)$. It turns out that the critical $u_{c}$-value is a monotonic function of $Z_{B}$ (or $Z_{A}$ ): when $Z_{A}>Z_{B}$ we find $u_{c}=0.2578$ for $Z_{A} \approx Z_{B}$ and $u_{c}=$ 0.2592 for $Z_{B}=0$; when $Z_{A}<Z_{B}$ we find $u_{c}=0.2550$ for $Z_{A}=0$ and $u_{c}=0.2578$ for $Z_{A} \approx Z_{B}$. Within these $u$ regions [white stripes in Fig. 1(b) and 1(c)] the PIE model is not applicable for two main reasons: (i) for $u \approx u_{c}$ the electrostatic energy differences are small and may be comparable to other nonelectrostatic contributions, and (ii) the ionic charges $Z_{A}$ and $Z_{B}$ are not known exactly in practice.

The databases used for testing.-In this Letter, we consider oxide spinels at low temperature that are: (i) stoichiometric, and (ii) cubic ( $F d 3 m$ space group) or approximately cubic ( $I 4_{1} /$ amd space group), for which $u$ can be defined and measured (11 out of 21 reported in Refs. [1,6]). This includes compounds with $A=B$ such as

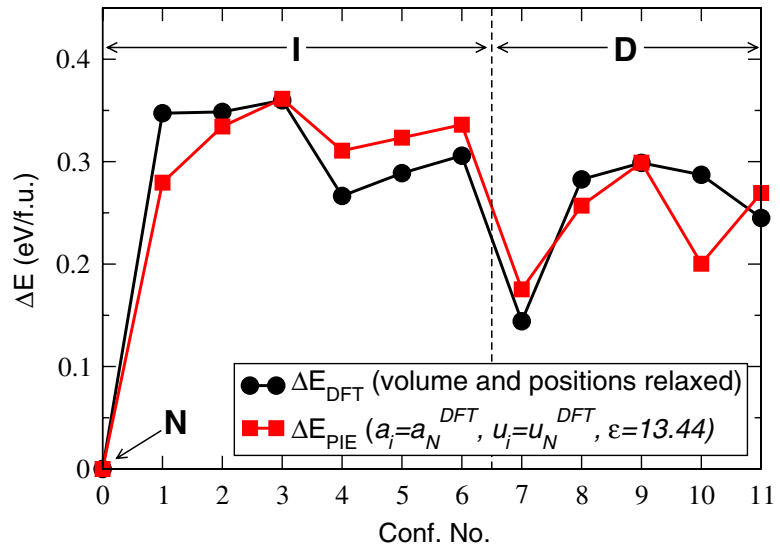

FIG. 2 (color online). Comparison of DFT and the PIE relative energies of six inverse $(I)$ and five dual $(D) \mathrm{Al}_{2} \mathrm{MgO}_{4}$ configurations. The PIE energies are computed using the formal ionic charges and a fixed set $a=8.15 \AA$ and $u=0.2639$ from the DFT-relaxed normal $\mathrm{Al}_{2} \mathrm{MgO}_{4}$ structure. The scaling factor $\varepsilon=$ 13.44 of the (PIE) is fitted to $\Delta E_{\mathrm{DFT}}$. 
TABLE I. List of compounds for which there is a disagreement between experimental (Refs. [1,22]), DFT and the PIE model $N / I$ assignments. Note that using $u_{\mathrm{DFT}}$ in place of $u_{\text {exp }}$ in the PIE model corrects 9 of 11 apparent errors. The DFT inverse vs normal energy difference $\Delta E_{I-N}^{\mathrm{DFT}}$ is also reported. See text for discussion of $\mathrm{Al}_{2} \mathrm{CdO}_{4}, \mathrm{In}_{2} \mathrm{MgO}_{4}$, and $\mathrm{In}_{2} \mathrm{ZnO}_{4}$.

\begin{tabular}{|c|c|c|c|c|c|c|c|}
\hline \multirow[b]{2}{*}{$\mathrm{A}_{2} \mathrm{BO}_{4}$} & \multicolumn{4}{|c|}{$N / I$ assignment } & \multirow[b]{2}{*}{$u_{\text {exp }}$} & \multirow[b]{2}{*}{$u_{\mathrm{DFT}}$} & \multirow{2}{*}{$\begin{array}{c}\Delta E_{I-N}^{\mathrm{DFT}} \\
\text { [eV/f.u.] }\end{array}$} \\
\hline & exp. & DFT & $\operatorname{PIE}\left(u_{\exp }\right)$ & $\operatorname{PIE}\left(u_{\mathrm{DFT}}\right)$ & & & \\
\hline \multicolumn{8}{|c|}{$Z_{A}>Z_{B}$} \\
\hline $\mathrm{Cr}_{2} \mathrm{HgO}_{4}$ & $N$ & $N$ & $I$ & $N$ & 0.2297 & 0.2719 & 2.14 \\
\hline $\mathrm{Ti}_{2} \mathrm{MgO}_{4}$ & $N$ & $N$ & $I$ & $N / I$ & 0.25 & 0.2580 & 0.57 \\
\hline $\mathrm{Ga}_{2} \mathrm{CuO}_{4}$ & $I$ & $I$ & $N$ & $I$ & 0.2595 & 0.2554 & -0.08 \\
\hline $\mathrm{Ga}_{2} \mathrm{NiO}_{4}$ & $I$ & $I$ & $N$ & $I$ & 0.262 & 0.2560 & -0.54 \\
\hline $\mathrm{Mn}_{2} \mathrm{NiO}_{4}$ & $I$ & $N$ & $N$ & $N$ & 0.2626 & 0.2605 & 0.22 \\
\hline $\mathrm{Sn}_{2} \mathrm{ZnO}_{4}$ & $I$ & $I$ & $N$ & $N$ & 0.2650 & 0.2670 & -1.27 \\
\hline $\mathrm{Al}_{2} \mathrm{CdO}_{4}$ & $I$ & $N$ & $N / I$ & $N$ & 0.259 & 0.2704 & 1.29 \\
\hline $\mathrm{In}_{2} \mathrm{MgO}_{4}$ & $I$ & $I$ & $I$ & $N$ & 0.2561 & 0.2598 & -0.06 \\
\hline $\mathrm{In}_{2} \mathrm{ZnO}_{4}$ & & $N$ & & $I$ & & 0.2553 & 0.26 \\
\hline \multicolumn{8}{|c|}{$Z_{A}<Z_{B}$} \\
\hline $\mathrm{Zn}_{2} \mathrm{PdO}_{4}$ & $I$ & $I$ & $N$ & $I$ & 0.25 & 0.2596 & -2.20 \\
\hline $\mathrm{Mg}_{2} \mathrm{PtO}_{4}$ & $I$ & $I$ & $N$ & $I$ & 0.25 & 0.2596 & -2.92 \\
\hline $\mathrm{Zn}_{2} \mathrm{VO}_{4}$ & $I$ & $I$ & $N$ & $I$ & 0.25 & 0.2604 & -0.25 \\
\hline $\mathrm{Fe}_{2} \mathrm{VO}_{4}$ & $I$ & $I$ & $N$ & $N / I$ & 0.253 & 0.2571 & -0.08 \\
\hline $\mathrm{Na}_{2} \mathrm{MoO}_{4}$ & $N$ & $N$ & $I$ & $N$ & 0.2617 & 0.2377 & 1.60 \\
\hline
\end{tabular}

$\mathrm{Co}_{2}^{3+} \mathrm{Co}^{2+} \mathrm{O}_{4}$ and $\mathrm{Fe}_{2}^{3+} \mathrm{Fe}^{2+} \mathrm{O}_{4}$. We do not consider alloys of spinels. In principle, spinels with $Z_{B}=0$ (e.g., lambda $\mathrm{MnO}_{2}$ ) could also be addressed, but they fall in the group of cation deficient spinel oxides which are not considered in this Letter. 117 such spinels have been characterized previously (Ref. [1]) and are listed in the supplementary material [16]. For 84 out of the 117 spinel oxides reported in the experimental compilation of Refs. [1,6] both the $u$ parameter and the $I$ vs $N$ preference have been measured. For 30 cases no $(a, u)$ values could be found in the literature and for 3 additional spinels, $\mathrm{Co}_{2} \mathrm{CuO}_{4}, \mathrm{Co}_{2} \mathrm{FeO}_{4}$ and $\mathrm{Fe}_{2} \mathrm{MoO}_{4}$ the reported $(a, u)$ correspond to very high degree of inversion $\lambda=0.5$ so they are close to be neither $N$ nor $I$. To further test our model we have augmented the experimental data set by 18 additional cases taken from DFT calculations of $(a, u)$ reported in Ref. [7] These are listed in [16] .

Delineating normal from inverse via the PIE model.We have placed all 84 oxide spinels in Fig. 1 according to their affiliation to one of the two classes $\left(Z_{A}>Z_{B}\right.$ or $Z_{A}<$ $Z_{B}$ ), measured $u_{\exp }$ and measured structural preference, with normal spinels shown in blue and inverse spinels in red. The association to one of the two classes is determined according to the formal oxidation states of the cations. In the cases when this cannot be done unambiguously (multivalent cations) we compare the most prevalent oxidation state [21] of each cation. Had the PIE model been completely successful, all of the red labels (inverse-preferring compounds) in Fig. 1(b) $\left(Z_{A}>Z_{B}\right)$ would have been to the left of the $u_{c}=0.2592$ vertical line and all the blue crosses (normal-preferring) to the right of the $u_{c}=0.2578$ vertical line (two compounds are within $0.2578<u<0.2592$ ). In reality, 6 compounds occur as apparent errors in Fig. 1(b).
Similarly, in Fig. 1(c) all of the red-labeled (inversepreferring compounds) would need to be to the right of the $u_{c}=0.2550$ vertical line, and all of the blue crosses (normal-preferring compounds) to the left of the $u_{c}=$ 0.2578 vertical line. In reality, five compounds occur as apparent errors in Fig. 1(c) (and six are found within the indeterminate region $0.2550<u<0.2578$ ). These errors are summarized in Table I.

Apparent disagreement with literature due to possibly incorrect input $u_{\exp }$ values.-Experimentally, measuring $u$ is no trivial matter since it requires a structural refinement. Hence, we compared measured $u_{\text {exp }}$ for all structures in Table I to $u_{\text {DFT }}$ as obtained from first principles totalenergy minimization. DFT reproduces accurately experimental geometric data, as shown for spinels in Ref. [7]. In the case of inverse spinels, we define $u$ as the least-square fit of the oxygen sublattice, relaxed by DFT, of the $P 4_{1} 22$ ordered inverse structure to the normal oxygen sublattice (parameterized by $u$ and $a$ ). $P 4_{1} 22$ is known to be the low temperature structure of many inverse spinel oxides $[8,9,23]$. All DFT calculations are done with the PBE exchange-correlation functional [24], within the projected augmented wave method [25] as implemented in VASP [26], with a $6 \times 6 \times 6$ Monkhorst-Pack k-point mesh [27] and a plane wave cutoff of $400 \mathrm{eV}$. In 10 compounds $\left(\mathrm{Cr}_{2} \mathrm{HgO}_{4}, \mathrm{Ti}_{2} \mathrm{MgO}_{4}, \mathrm{Ga}_{2} \mathrm{CuO}_{4}, \mathrm{Ga}_{2} \mathrm{NiO}_{4}, \mathrm{Sn}_{2} \mathrm{ZnO}_{4}\right.$, $\mathrm{Zn}_{2} \mathrm{PdO}_{4}, \mathrm{Mg}_{2} \mathrm{PtO}_{4}, \mathrm{Zn}_{2} \mathrm{VO}_{4}, \mathrm{Fe}_{2} \mathrm{VO}_{4}$, and $\mathrm{Na}_{2} \mathrm{MoO}_{4}$ ) the $N$ vs $I$ DFT predictions and experimental assignments agree but the values of $u$ do not (see Table I). Using the theoretical $u_{\mathrm{DFT}}$ as input to the PIE model corrects the previous disagreement with experiment for 9 out of 11 compounds. We are thus inclined to consider these 9 cases as valid PIE predictions, not errors, and suggest that the 
measured $u$ values for these should perhaps be revisited experimentally.

Apparent disagreement with literature due to possibly incorrect experimental $N$ vs I assignment.-DFT totalenergy calculations disagree with the experimental $N$ versus $I$ assignment for two compounds, $\mathrm{Mn}_{2} \mathrm{NiO}_{4}$ and $\mathrm{Al}_{2} \mathrm{CdO}_{4}$. Whether using experimental or theoretical $u$ values as input, the PIE model predicts, as does direct DFT total energy, a preference for the normal structure where experiment [1] gives $I$. Finally, although $\mathrm{Al}_{2} \mathrm{CdO}_{4}$ is not a PIE error per se, there is doubt in literature [7] that its structure is incorrectly assigned to be inverse. Our DFT total-energy calculations and PIE based on $u_{\mathrm{DFT}}$ both predict the normal structure as the ground state. Thus, we are inclined to suggest that the $\mathrm{Mn}_{2} \mathrm{NiO}_{4}$ and $\mathrm{Al}_{2} \mathrm{CdO}_{4}$ $N$ vs. I experimental assignment to be reexamined.

Remaining real errors of the PIE model.-As shown in Table I, $\mathrm{Sn}_{2} \mathrm{ZnO}_{4}$ represents a real error in that DFT agrees with experiment about the $u$ value and about the structure (I), yet the PIE model predicts the $N$ structure in conflict with both experiment and DFT. Since the Sn appears only as a $\mathrm{Sn}^{+2}$ or $\mathrm{Sn}^{+4}$ cation (never $\mathrm{Sn}^{3+}$ ), one possible explanation is that all tetrahedral $\mathrm{Sn}$ are $\mathrm{Sn}^{+2}$ and all octahedral $\mathrm{Sn}$ are $\mathrm{Sn}^{+4}$. In this case the compound would be chemically inverse $Z_{A}>Z_{B}$ spinel oxide, but according to the charge it would still be a normal spinel with mixed $\mathrm{Sn}^{+4}$ and $\mathrm{Zn}^{+2}$ cations on octahedral sites. We do not expect that the PIE model is able to capture such situations so we count $\mathrm{Sn}_{2} \mathrm{ZnO}_{4}$ as a real PIE error. Failure of the PIE model is also expected for systems with distributed charge density such as spinel sulfides or selenides.

Application of the PIE model to other compounds using only DFT input.-We also applied our PIE model taking $u_{\text {DFT }}$ from Ref. [7] as input. The PIE model correctly predicts the structure of all but two of 18 spinel oxides (listed also in [16]): (i) $\mathrm{In}_{2} \mathrm{MgO}_{4}$ (DFT inverse but PIE normal), and (ii) $\mathrm{In}_{2} \mathrm{ZnO}_{4}$ (DFT normal, but PIE inverse). Since $\mathrm{In}_{2} \mathrm{MgO}_{4}$ is not a PIE error when $u_{\text {exp }}$ is used as the input and the calculated $u_{\mathrm{DFT}}=0.2598$ is very close to the borderline $(0.2592)$ and the inverse-normal DFT relative energy is rather small (only $-0.06 \mathrm{eV} /$ f.u.) [7] we do not consider this compound as real error. On the other hand, the structural preference of $\mathrm{In}_{2} \mathrm{ZnO}_{4}$ is not known experimentally, to the best of our knowledge $[1,6]$. However, DFT total energies clearly predicts a normal structure $(0.26 \mathrm{eV} /$ f.u. $)$, when the PIE model clearly predicts that it is inverse when $u_{\mathrm{DFT}}=0.2553$ is taken as the input. As such, we hold this spinel compound to be one of very few not amenable to an electrostatic interpretation.

The results presented clearly show that, contrary to the widely accepted opinion, main interactions in spinel oxides are of the electrostatic origin. Our model shows that the preference of spinel oxide compounds for inverse or normal cationic distribution can be successfully accounted for with $\sim 98 \%$ overall success rate (only two real errors out of $84+18=102$ compounds). The main accomplishment of this work is that it offers an effortless method for predicting the preference of a new spinel oxide compounds for the inverse or normal cationic distribution. As it is shown the model also identifies cases that are worth of experimental reexamination.

Research supported by the U.S. Department of Energy, Office of Basic Sciences, Division of Materials Sciences and Engineering, Energy Frontier Research Centers, under Grant No. DE-AC36-08GO28308 to NREL.

*alex.zunger@nrel.gov

[1] X. Zhang and A. Zunger, Adv. Funct. Mater. 20, 1944 (2010).

[2] N. W. Grimes, Physics in Technology 6, 22 (1975).

[3] M. Dekkers, G. Rijnders, and D. H. A. Blank, Appl. Phys. Lett. 90, 021903 (2007).

[4] J. Akimoto et al., J. Solid State Chem. 96, 446 (1992).

[5] Y. Yamasaki et al., Phys. Rev. Lett. 96, 207204 (2006).

[6] R. J. Hill, J. R. Craig, and G. V. Gibbs, Phys. Chem. Miner. 4, 317 (1979).

[7] S.H. Wei and S.B. Zhang, Phys. Rev. B 63, 045112 (2001).

[8] A. Seko et al., Phys. Rev. B 73, 184117 (2006).

[9] A. Seko, F. Oba, and I. Tanaka, Phys. Rev. B 81, 054114 (2010).

[10] E. J. W. Verwey, F. de Boer, and J. H. van Santen, J. Chem. Phys. 16, 1091 (1948).

[11] J. K. Burdett, G. D. Price, and S. L. Price, J. Am. Chem. Soc. 104, 92 (1982).

[12] O'Neil and A. Navrotsky, Am. Mineral. 68, 181 (1983).

[13] G. Blasse, Philips Res. Rep., Suppl., No. 3 (1964).

[14] K. E. Sickafus and J. M. Willis, J. Am. Ceram. Soc. 82, 3279 (1999).

[15] L. Bellaiche and D. Vanderbilt, Phys. Rev. Lett. 81, 1318 (1998).

[16] See supplmentary material at http://link.aps.org/ supplemental/10.1103/PhysRevLett.105.075501.

[17] P. P. Ewald, Ann. Phys. (Leipzig) 369, 253 (1921).

[18] J.E. Bernard and A. Zunger, Phys. Rev. B 36, 3199 (1987).

[19] L. Hermans, J. Weenk, and W. van Gool, Zeitschrift für Physikalische Chemie Neue Folge 88, 15 (1974).

[20] P. Thompson and N.W. Grimes, Philos. Mag. 36, 501 (1977).

[21] N. N. Greenwood and A. Earnshow, Chemistry of the Elements (Butterworth-Heinemann, Oxford; Boston, 1997), 2nd ed.

[22] Inorganic Chemistry Structural Database, URL http:// www.fiz-karlsruhe.de/icsd.html.

[23] B. A. Wechsler and A. Navrotsky, J. Solid State Chem. 55, 165 (1984).

[24] J. P. Perdew, K. Burke, and M. Ernzerhof, Phys. Rev. Lett. 77, 3865 (1996).

[25] P. E. Blöchl, Phys. Rev. B 50, 17953 (1994).

[26] G. Kresse and J. Furthmüller, Comput. Mater. Sci. 6, 15 (1996).

[27] H. J. Monkhorst and J. D. Pack, Phys. Rev. B 13, 5188 (1976). 